\title{
The kaehlerian structures and reproducing kernels
}

\author{
by Anna Krok and Tomasz Mazur (Radom)
}

\begin{abstract}
It is shown that one can define a Hilbert space structure over a kaehlerian manifold with global potential in a natural way.
\end{abstract}

Introduction. S. Bergman introduced and developed some methods of functional analysis and differential geometry in the theory of several complex variables $[2,3]$. In this approach important role is played by the Hilbert space $L^{2} H(D)$ of all functions which are holomorphic and Lebesgue square integrable on a domain $D \subset \mathbb{C}^{N}$. The evaluation functional

$$
\chi_{z}^{*}: L^{2} H(D) \rightarrow \mathbb{C}, \quad \chi_{z}^{*}(f)=f(z),
$$

is continuous and can be represented by $\chi_{z} \in L^{2} H(D)$ as follows:

$$
f(z)=\left(f, \chi_{z}\right)
$$

(for details see $[2,10,12])$. The well-known Bergman function $[2,12]$

$$
K_{D}(z, w)=\left(\chi_{w}, \chi_{z}\right)
$$

generates a geometric structure on $D$, given by a tensor $g$ of the form

$$
g(z)=\sum_{i, j=1}^{N}\left(g_{i \bar{j}}(z) d z_{i} \otimes d \bar{z}_{j}+g_{\overline{i j}}(z) d \bar{z}_{i} \otimes d z_{j}\right)
$$

where

$$
g_{i \bar{j}}(z):=\partial^{2} \log K_{D}(z, z) / \partial z_{i} \partial \bar{z}_{j} .
$$

The tensor $g$ defines a kaehlerian structure on every bounded domain $D \subset$ $\mathbb{C}^{N}[2,12]$.

The situation described above was the starting point of fruitful investigations exhibiting fine connections between different branches of mathematics: spectral theory of operators in Hilbert space [8, 10], ergodic theory

1991 Mathematics Subject Classification: Primary 58A46, 58A32.

Key words and phrases: kaehlerian manifold, kaehlerian potential, positive definite function, Bergman function, reproducing kernel. 
[5], group representations [7] and mathematical physics [6, 8, 9]. The link between pseudo-riemannian geometry and Hilbert space methods is very interesting from mathematical-physics point of view. Both these subjects are tools of large parts of physics: general relativity and quantum mechanics. In this context, we try to explain that the notions of kaehlerian manifold and of a Hilbert space with a reproducing kernel are very strongly related. In [9], the problem of when a reproducing kernel in a Hilbert space of functions $f: X \rightarrow \mathbb{C}$ generates a kaehlerian structure on $X$ is solved. In the present paper we consider a case when a kaehlerian potential produces a reproducing kernel in some Hilbert space.

1. Kaehlerian manifolds, reproducing kernels and positive definite functions. We recall the basic notions which will be used in this paper.

A complex manifold $\mathbf{M}$ with a tensor $g$ is kaehlerian if:

1) $g$ is a riemannian metric tensor on $\mathbf{M}$ as a real manifold,

2) the $\mathbb{C}$-linear extension of $g$ to the complex tangent bundle TM is invariant w.r.t. to the operator $J$ of complex structure,

3) the exterior form

$$
\omega(Z, W):=g(J Z, W)
$$

is closed i.e. $d \omega=0(Z, W$ are sections of $\mathbf{T M})$.

A complex manifold $\mathbf{M}$ is kaehlerian if and only if there exists a locally defined complex-valued $C^{\infty}$-function $F$ on $\mathbf{M}$ such that

$$
\omega=\partial \bar{\partial}(F-\bar{F})
$$

(for details see [4], pp. 59-60). The function

$$
p(z):=-i(F(z)-\overline{F(z)}), \quad z \in \mathbf{M},
$$

is called the kaehlerian potential on $\mathbf{M}$.

Let $\mathbf{X}$ be an arbitrary set. A non-zero function $k: \mathbf{X} \times \mathbf{X} \rightarrow \mathbb{C}$ is positive definite if for any $t_{1}, \ldots, t_{n} \in \mathbf{X}$ and any $c_{1}, \ldots, c_{n} \in \mathbb{C}$

(a) $\sum_{i, j=1}^{n} c_{i} \bar{c}_{j} k\left(t_{i}, t_{j}\right) \geq 0$,

(b) $k\left(t_{i}, t_{j}\right)=\overline{k\left(t_{j}, t_{i}\right)}$.

Let $(H,()$,$) be a Hilbert space of complex functions defined on \mathbf{X}$. A function $K: \mathbf{X} \times \mathbf{X} \rightarrow \mathbb{C}$ is called a reproducing kernel for $(H,(\cdot, \cdot))$ if:

(a) $K(\cdot, y) \in H$ for each $y \in \mathbf{X}$,

(b) $h(y)=(h, \mathbf{K}(\cdot, y))$ for each $h \in H$ and $y \in \mathbf{X}$.

1.1. Remark. The Bergman function (0.1) is a reproducing kernel in $L^{2} H(D)$. 
1.2. Remark. Every reproducing kernel is positive definite.

1.3. TheOREM (Aronszajn). A Hilbert space $(H,(\cdot, \cdot))$ has a reproducing kernel if and only if for any $y \in \mathbf{X}$ there exists a constant $a(y)$ such that for any $h \in H$

$$
|h(y)| \leq a(y)\|h\| .
$$

2. How does a kaehlerian potential produce a reproducing kernel? The main purpose of this paper is to prove the following

2.1. THEOREM. Let $\mathbf{M}$ be a kaehlerian manifold with potential $p$ of the form (1.3). If the function $F$ (and so $p$ ) is defined globally on $\mathbf{M}$, then

$$
K(z, w):=e^{-i(F(z)-\overline{F(w)})}, \quad z, w \in \mathbf{M},
$$

is a reproducing kernel in some Hilbert space.

Proof. First we will show that $K$ is positive definite; then we use a method due to N. Aronszajn [1] (see also [11]) to construct the desired space. Indeed,

$$
\begin{gathered}
\overline{K\left(z_{j}, z_{i}\right)}=e^{\overline{-i\left(F\left(z_{j}\right)-\overline{F\left(z_{i}\right)}\right.}}=e^{-i\left(F\left(z_{i}\right)-\overline{F\left(z_{j}\right)}\right)}=K\left(z_{i}, z_{j}\right) \\
\sum_{i, j=1}^{n} c_{i} \bar{c}_{j} K\left(z_{i}, z_{j}\right)=\sum_{i, j=1}^{n} c_{i} \bar{c}_{j} e^{-i\left(F\left(z_{i}\right)-\overline{F\left(z_{j}\right)}\right)} \\
=\left(\sum_{i=1}^{n} c_{i} e^{-i F\left(z_{i}\right)}\right)\left(\sum_{j=1}^{n} \bar{c}_{j} e^{\overline{-i F\left(z_{j}\right)}}\right)=\left|\sum_{i=1}^{n} c_{i} e^{-i F\left(z_{i}\right)}\right|^{2} \geq 0 .
\end{gathered}
$$

Set

$$
\begin{array}{r}
H_{0}:=\left\{f: \mathbf{M} \rightarrow \mathbb{C} ; f(z)=\sum_{i=1}^{n} a_{i} K\left(z, t_{i}\right), a_{i} \in \mathbb{C}, z, t_{i} \in \mathbf{M}, i=1 \ldots n\right. \\
n=1,2, \ldots\} .
\end{array}
$$

If $f(\cdot)=\sum_{i=1}^{n_{1}} a_{i} K\left(\cdot, t_{i}\right)$ and $g(\cdot)=\sum_{j=1}^{n_{2}} b_{j} K\left(\cdot, \tau_{j}\right)$ set

$$
(f, g)_{0}=\sum_{i, j=1}^{n} a_{i} \bar{b}_{j} \overline{K\left(\tau_{j}, t_{i}\right)}, \quad n=\min \left(n_{1}, n_{2}\right) .
$$

Clearly, $(\cdot, \cdot)_{0}$ is a scalar product in $H_{0}$. Let $(H(K),(\cdot, \cdot))$ be the completion of $\left(H_{0},(\cdot, \cdot)_{0}\right)$. Then $(H(K),(\cdot, \cdot))$ is a Hilbert space for which $K$ is a reproducing kernel.

3. Examples. 1. Let $\mathbf{M}=D$ be a bounded domain in $\mathbb{C}^{N}$. In this case the kaehlerian potential (1.2) has the form $p(z)=\log K_{D}(z, z)$. Then $(2.1)$ 
is exactly the Bergman function, and moreover $H\left(K_{D}\right)=L^{2} H(D)$, up to isomorphism.

2. Let $\mathbf{M}=\mathbb{C}^{1}$. Consider the geometry given by the tensor

$$
g(z)=1 d z \otimes d \bar{z}+1 d \bar{z} \otimes d z .
$$

$g$ describes a kaehlerian geometry on the plane, which is in fact euclidean in the real sense. In this case (see [9]) $K_{\mathbb{C}}(z, w)=e^{z \bar{w}}$ and $H\left(K_{\mathbb{C}}\right)$ is the well-known Fock space.

\section{References}

[1] N. Aronszajn, Theory of reproducing kernels, Trans. Amer. Math. Soc. 68 (1956), $337-404$.

[2] S. Bergman, The Kernel Function and Conformal Mapping, 2nd ed., Math. Surveys 5, Amer. Math. Soc., 1970.

[3] —,Über die Kernfunktion eines Bereiches und ihr Verhalten am Rande, J. Reine Angew. Math. 169 (1933), 1-42.

[4] S. Chern, Complex Manifolds without Potential Theory, 2nd ed., Springer, 1978.

[5] W. Chojnacki, On some holomorphic dynamical systems, Quart. J. Math. Oxford Ser. (2) 39 (1988), 159-172.

[6] S. Janson, J. Peetre and R. Rochberg, Hankel forms and the Fock space, Rev. Mat. Iberoamericana 3 (1987), 61-138.

[7] S. Kobayashi, On the automorphism group of a homogeneous complex manifold, Proc. Amer. Math. Soc. 12 (3) (1961), 359-361.

[8] T. Mazur, Canonical isometry on weighted Bergman spaces, Pacific J. Math. 136 (2) (1989), 303-310.

[9] - On the complex manifolds of Bergman type, preprint.

[10] T. Mazur and M. Skwarczyński, Spectral properties of holomorphic automorphism with fixed point, Glasgow Math. J. 28 (1986), 25-30.

[11] W. Mlak, Introduction to Hilbert Space Theory, PWN, Warszawa 1991.

[12] M. Skwarczyński, Biholomorphic invariants related to the Bergman functions, Dissertationes Math. 173 (1980).

DEPARTMENT OF MATHEMATICS

TECHNICAL UNIVERSITY OF RADOM

MALCZEWSKIEGO 29

26-600 RADOM, POLAND 